\title{
Drag optimization for axisymmetric afterbodies with jet plume using computational fluid dynamics
}

\author{
Masoud Mirzaei* $\quad$ Sina Arabi*
}

(Received 20 November 2004, revised 19 September 2005)

\begin{abstract}
A supersonic turbulent flow over an axisymmetric body is numerically studied using the Navier-Stokes equations in conjunction with the $k$ - $\epsilon$ turbulence model. Numerical calculations of total body drag (including wave drag, skin friction drag, and base drag) were carried out for a boat tailed projectile with and without base-bleed. Surface pressures and viscous layer profiles are in good agreement with experimental data. Golden Section optimization was adopted to optimize the body boat tail. This optimization may be used as a tool for preliminarily design of axisymmetric boat tailed bodies.
\end{abstract}

${ }^{*}$ Mechanical Engineering Department, K. N. Toosi University of Technology, Tehran, IRAN. mailto:mirzaei@kntu.ac.ir

See http://anziamj.austms.org.au/V46/CTAC2004/Mirz for this article, (C) Austral. Mathematical Soc. 2005. Published October 14, 2005. ISSN 1446-8735 


\section{Contents}

1 Introduction

C1070

2 Governing equations

C1072

3 Numerical formulation

C1073

4 Optimization techniques

C1074

4.1 Aspects of drag optimization . . . . . . . . . . . . C1074

5 Results

C1076

5.1 Drag Optimization

C1079

6 Conclusions

C1083

References

C1083

\section{Introduction}

The aerodynamic design of a supersonic flying vehicle has a major role in overall design of such vehicles. One of the most important restrictions on the aerodynamic performance of a flying object is drag force. Wave drag, skin friction drag, and base drag are three main components of total drag force. Predictions of accurate values for these drag components and thus having valid tools for designing proposes, are very difficult. Specially, accurate prediction of base drag has long eluded the practicing engineer. For power-off or no base bleed conditions, base drag may even reach $75 \%$ of the total drag, but for power-on (or with base bleed) conditions, a considerable reduction of this drag component occurrs depending on the base diameter and base bleed conditions. Moreover, the effects of a lot of parameters such as Mach number, Reynolds number, the structure of the recirculation zones, 


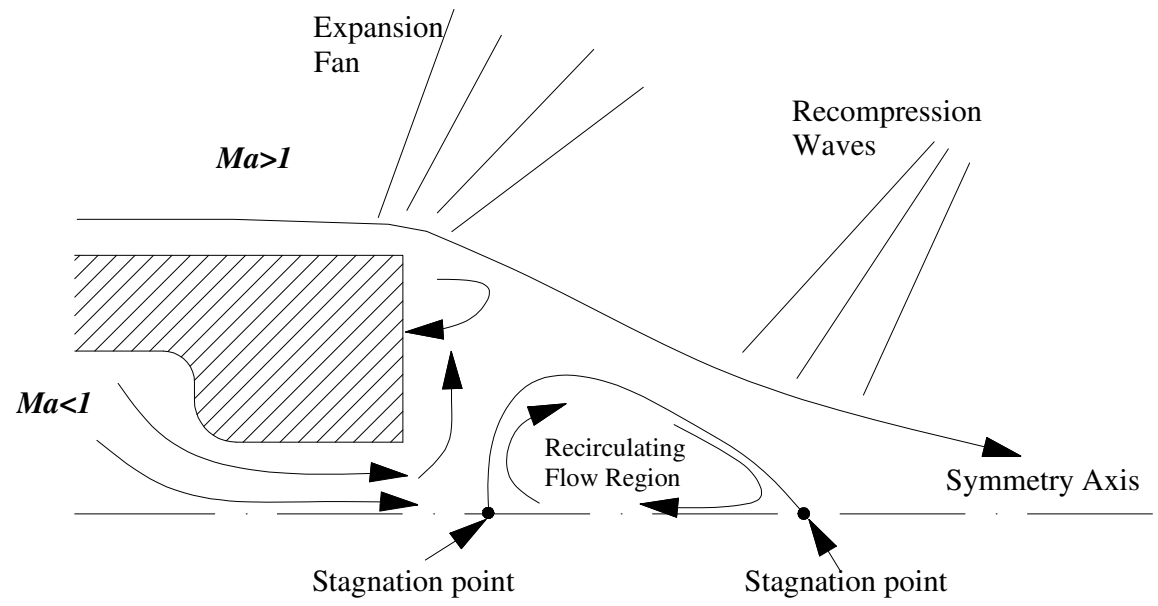

Figure 1: Flow field behind the base of a projectile at low base-bleed rate.

presence of boat tails and fins, cause difficulties in the accurate prediction of the base drag component. Figure 1 shows the basic structure of the flow field right behind the base of a projectile with base-bleed at a low base-bleed rate. See that the interaction between the shock-expansion and the recirculation zone results in a complex flow field behind the base. These complexities and the difficulties associated with accurately predicting the flow patterns, led researchers to utilize various semi-empirical prediction methods which were valuable but very limited in their application [12].

The advances in computer hardware and also improvement in computational fluid dynamics led researchers to solve the Navier-Stokes equations for simulation the flow field around base $[2,3,4,5,6,7,8,10,11,12,14]$. Most such work was carried out for flying bodies with no plume at the base (power off or no base bleed conditions). The present work deals with the calculation of drag components, especially the base drag component, in the presence of base bleed. Computations are carried out for entire flow field over an axisymmetric body using the Navier-Stokes equations in conjunction with the 
$k-\epsilon$ turbulence model equations. This turbulence model has the capability of predicting the base-bleed behavior and recalculating the flow in the remaining base region. This model has been used previously by Cumming et al. [1] for a similar problem.

The next step is to begin using these computations as a practical preliminary design tool. The goal of this research is to use the solution of the Navier-Stokes equations over an axisymmetric boattailed body with base bleed in the determination of the geometry for minimum total drag. As these types of applications are improved and extended, the designer will have a valuable tool to go along with wind tunnel testing as new configurations are developed for flying vehicles. The main feature of this work that makes it different from others is the inclusion of both base bleed and boattailing simultaneously in drag optimization of a supersonic projectile.

\section{Governing equations}

Because of complexities of the flow field, for simulation of such flow, the full Navier-Stokes are used as governing equations. The conservative form of these equations, the continuity equation and also the $k-\epsilon$ turbulence model equations for axisymmetric geometry are

$$
\frac{\partial U}{\partial t}+\frac{\partial E}{\partial r}+\frac{\partial F}{\partial z}+H=0,
$$

where the vectors

$$
U=\left[\begin{array}{c}
\bar{\rho} \\
\bar{\rho} \hat{u}_{r} \\
\bar{\rho} \hat{v}_{z} \\
\bar{\rho} \hat{\mathrm{E}} \\
\bar{\rho} \hat{k} \\
\bar{\rho} \hat{\varepsilon}
\end{array}\right], \quad E=r\left[\begin{array}{c}
\bar{\rho} \hat{u}_{r} \\
\bar{\rho} \hat{u}_{r} \hat{u}_{r}+\bar{p}-\bar{\tau}_{r r} \\
\bar{\rho} \hat{u}_{z} \hat{u}_{r}-\bar{\tau}_{z r} \\
\bar{\rho} \hat{u}_{r}(\bar{\rho} \hat{\mathrm{E}}+\bar{p})+\bar{q}_{r}-\hat{u}_{r} \bar{r}_{r r}-\hat{u}_{r} \bar{\tau}_{z r} \\
\bar{\rho} \hat{u}_{r} \hat{k}-\left(\bar{\mu}+\frac{\mu_{t}}{\sigma_{k}}\right) \frac{\partial \hat{k}}{\partial r} \\
\bar{\rho} \hat{u}_{r} \epsilon-\left(\bar{\mu}+\frac{\mu t}{\sigma_{k}}\right) \frac{\partial \epsilon}{\partial r}
\end{array}\right],
$$




$$
F=r\left[\begin{array}{c}
\bar{\rho} \hat{u}_{z} \\
\bar{\rho} \hat{u}_{r} \hat{u}_{z}+\bar{p}-\bar{\tau}_{r z} \\
\bar{\rho} \hat{u}_{z} \hat{u}_{z}-\bar{\tau}_{z z} \\
\bar{\rho} \hat{u}_{z}(\bar{\rho} \hat{\mathrm{E}}+\bar{p})+\bar{q}_{z}-\hat{u}_{r} \bar{\tau}_{r z}-\hat{u}_{z} \bar{\tau}_{z z} \\
\bar{\rho} \hat{u}_{z} \hat{k}-\left(\bar{\mu}+\frac{\mu_{t}}{\sigma_{k}}\right) \frac{\partial \hat{k}}{\partial z} \\
\bar{\rho} \hat{u}_{z} \epsilon-\left(\bar{\mu}+\frac{\mu_{t}}{\sigma_{k}}\right) \frac{\partial \epsilon}{\partial z}
\end{array}\right], \quad H=\left[\begin{array}{c}
0 \\
-\bar{p}+\bar{\tau}_{\theta \theta} \\
0 \\
0 \\
r(P-\bar{\rho} \epsilon) \\
\frac{r \epsilon}{\hat{k}}\left(C_{1} P-C_{2} \bar{\rho} \epsilon\right)
\end{array}\right] .
$$

The shear stress, the heat flux, the turbulence production, and the constants are defined in [1]. The above set of equations contains six equations with seven unknowns; to close this set we need an extra equation, the equation of state for the fluid (which is air):

$$
\bar{p}=\bar{\rho} R \hat{T} .
$$

\section{$3 \quad$ Numerical formulation}

The finite volume technique is adopted for spatial discretization of the governing equations. After integration of equation (1) over triangular control volume $j$, we have

$$
\frac{d \bar{U}_{j}}{d t} A_{j}+\sum_{i=1}^{3}\left(\bar{E}_{n_{i}}+\bar{F}_{n_{i}}\right) \Delta s_{i}+\bar{H}_{j} A_{j}=0 .
$$

Here $\bar{U}_{j}$ and $\bar{H}_{j}$ are numerical approximations to $U$ and $H$ on the control volume, $\bar{E}_{n_{i}}$ and $\bar{F}_{n_{i}}$ are approximations to the normal components of $E$ and $F$ on edge $i$ of the control volume, $\Delta s_{i}$ is the length of edge $i$, and $A_{j}$ denotes the area of the control volume $j$. The viscous fluxes are calculated using a central difference approximation which is compatible with the nature of these fluxes. The Kinetic Flux Vector Splitting algorithm is chosen to approximate the inviscid fluxes. This algorithm is based on the correlation between the Boltzmann Equation and the Euler equations. More details of the algorithm may be found in [9]. 


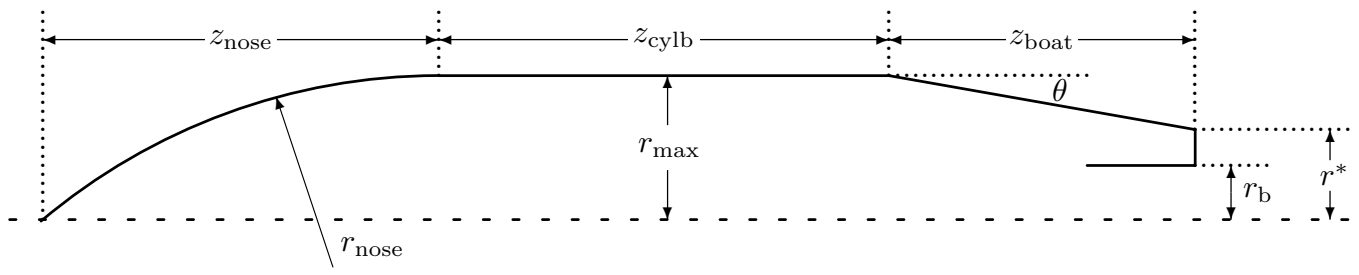

Figure 2: Geometry of the projectile.

The resulting system of ordinary differential equations is solved using a fourth-order Runge-Kutta method.

\section{Optimization techniques}

The goal of any mathematical optimization is to extremize (minimize or maximize) some objective function, $f$. The objective function must be expressible as a single value for the optimization technique to operate, but $f$ may be a function of several design variables, $x_{1}, x_{2}, \ldots, x_{N}$, which are to be selected in order to optimize $f$. In principle, as many design variables, $N$, as a desired may be used. However, in practice the number of design variables is kept at a minimum since the optimization efficiency and computational time for most algorithms varies proportionally as $N^{2}$.

\subsection{Aspects of drag optimization}

Before choosing minimization algorithm, the general aspects of the problem to be solved must be defined. By evaluating the various minimization techniques with the specific problem in mind, a good choice of technique can be made. The goal of this computation is to predict the total drag of an axisymmetric body at zero angle of attack in compressible turbulent flow. The 
drag will be determined as the sum of the pressure drag, skin friction drag, and base drag. The only design variable, which will be considered for optimization, is the boattail angle, $\theta$ (see Figure 2). The boattail length, $z_{\text {boat }}$, will not be considered as an additional design variable since experiments have shown that body drag decreases approximately linearly with increasing boattail length [1]. An optimization of boattail length would always result in the largest considered value of boattail length. As boattail angle varies, the drag components due to pressure and skin friction will vary, yielding a minimum value at some boattail angle within prescribed constraints. The goal of the optimization is to obtain the body shape, which minimizes drag for a given geometry type, therefore the objective function is the total drag. Since only one design variable is being used, it will be helpful to know a priori the general relationship of drag with the boattail angle. Since this case represents a single dimension design space, which is known to be unimodal, a search method can be adopted in order to efficiently obtain the minimum objective function, with the overall goal being to minimize total computation time (both the time of operating the search method and the time of calculating the objective function).

Based on this knowledge of the design space, a reasonable search method would be an elimination technique, especially since all of the gradient methods would require calculation of the derivative of $f$, a very time consuming operation (each computation of $f$ will require one converged solution of the CFD code, so minimizing the number of evaluations of $f$ is paramount to overall computational efficiency). The Golden Section method [1] is used for this optimization due to its robust nature and capability to locate a minimum without using derivatives. 

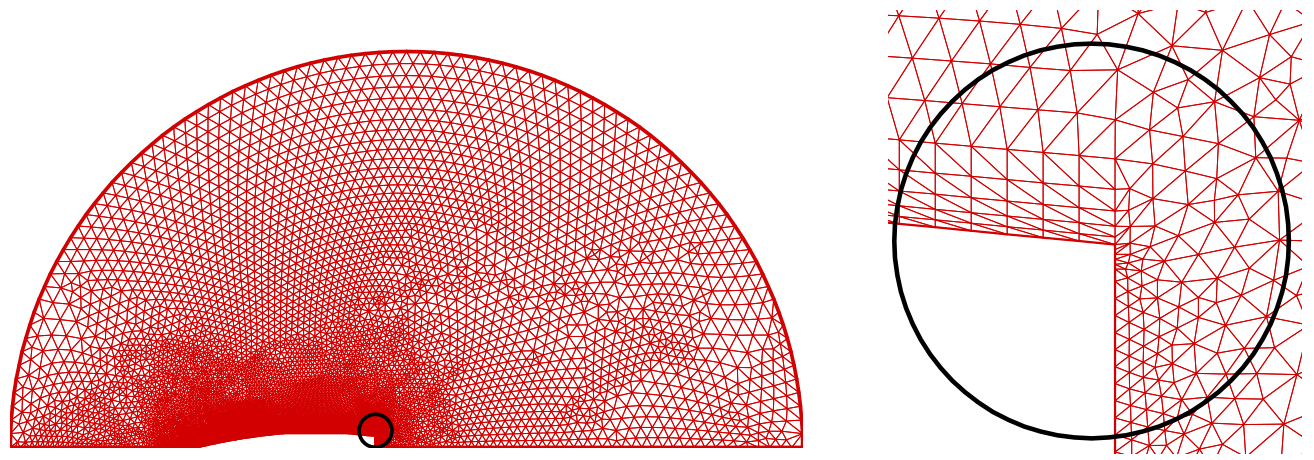

FiguRE 3: Grid point distribution.

\section{Results}

The geometry of the problem is shown in Figure 2. This figure shows an axisymmetric projectile with secant-ogive forebody/cylindrical afterbody, with and without boattail and base bleed. The length of the body is six diameters $(D)$ of the body and $r_{\text {nose }}=18.88 D, z_{\text {nose }}=3.0 D, z_{\text {cylb }}=2.0 D$, $z_{\text {boat }}=1.0 D, r^{*}=0.38 D, r_{\max }=0.5 D$, and $r_{\mathrm{b}}=0.15 D$. The body diameter is $D=5.715 \mathrm{~cm}$. The boat tail angle $\theta$ is the optimization parameter and for the $\theta=0^{\circ}$ and $\theta=7^{\circ}$ cases experimental results are available. Several tests were conducted to measure both surface pressure and boundary layer quantities at Mach 3.0. These tests were carried out at a Reynolds number (based on the body length) of $6.5 \times 10^{6}$.

In order to have grid independent numerical results a fine computational grid is required. Figure 3 shows one of the grids used for the flow field computations. This grid contains 30571 points and 60742 computational cells (control volumes) and gives grid independent results. This is shown in Figure 4 where the drag coefficient is plotted against boattail angle for three different grid sizes, and the numerical results reported in [1] are also shown. This plot not only shows the grid independency of the results for 60742 computa- 


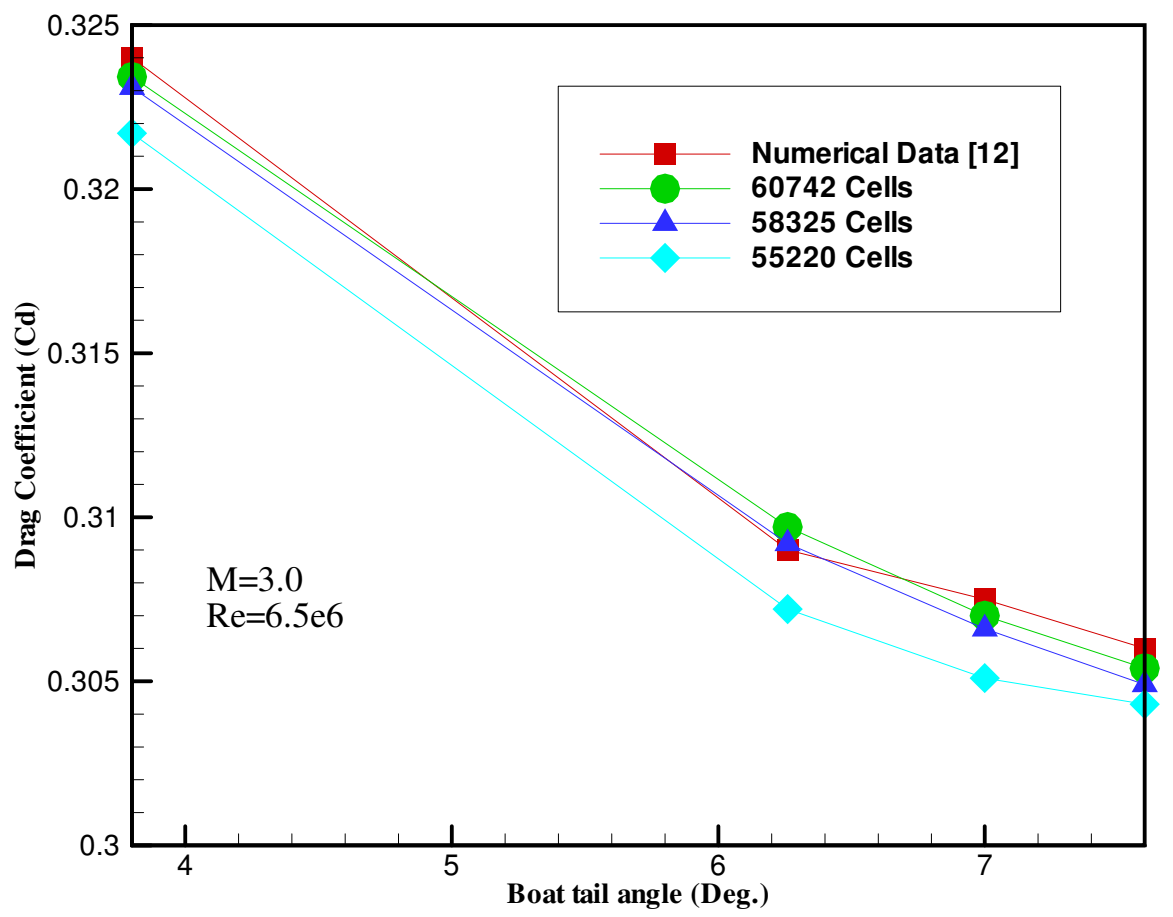

FiguRE 4: Grid independence and validation of the results.

tional cells, but also validates the present calculations. Maximum deviation of the present results (with 60742 cells) from the results of [1] is less than $1 \%$. The computations were performed using a Pentium IV $2.8 \mathrm{GHz}$ personal computer. The CPU time for 30571 computational points was 850 seconds, and the required memory for these computations was $120 \mathrm{Mb}$.

Before using the numerical code for boattail angle optimization for minimizing overall drag, the result of the code should be validated against experimental data.

The primary results for which test data are available are at free stream 


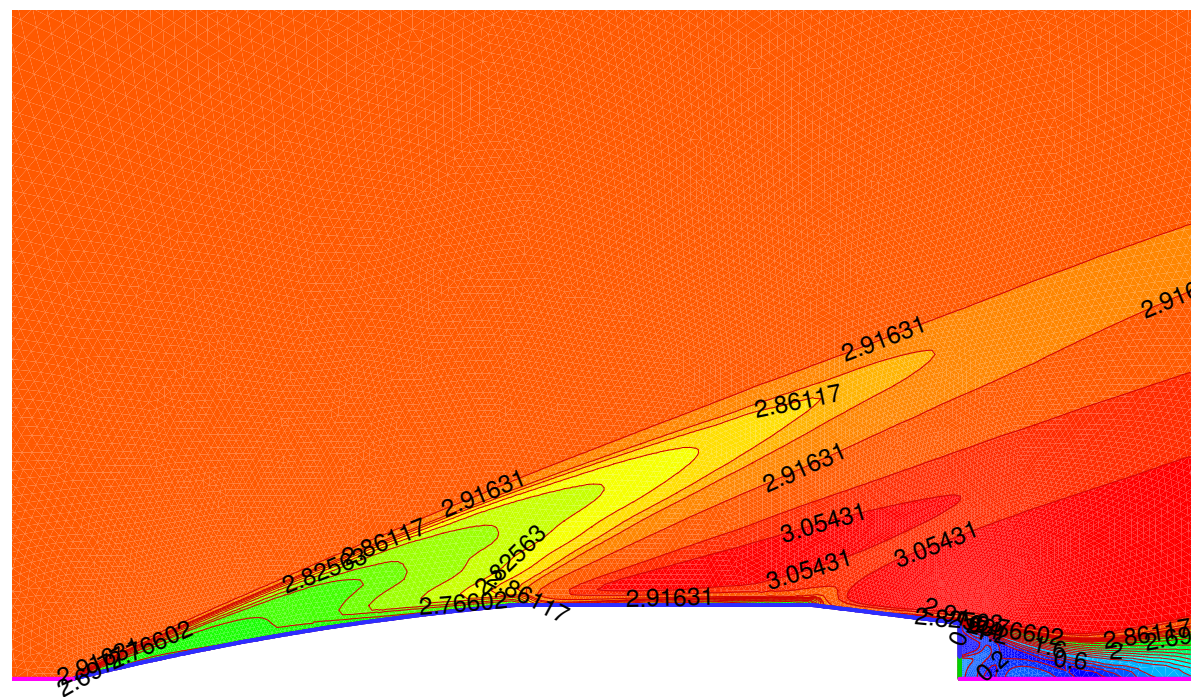

Figure 5: Mach contours around the projectile at $M=3.0$.

Mach number of 3.0 and a Reynolds number of $6.5 \times 10^{6}$. Data are available for boattail angles $\theta=0^{\circ}$ and $\theta=7^{\circ}$. General features of the flow field must be examined in order to gain confidence in the solution. In particular, there should be an attached oblique shock wave. Figure 5 shows Mach contours over the body for the free stream condition of $M=3.0$. See that the shocks and expansions are captured clearly.

The flow pattern at the base of the body is shown in Figure 6. This figure shows that the interaction of base bleed flow and the external flow produces two recirculation zones. These recirculation zones influence the base drag force. This flow pattern was shown schematically in Figure 1. In Figure 7, calculated boundary layer velocity profiles for the 3 axial locations $z / D=3.33,4.44$ and 5.56 are compared with experimental data reported in [15]. Profiles show that the turbulence model produces reasonable results in the viscous layer. Since the main results required of the code are the gross forces acting on the body, it appears that the turbulence model is doing an 


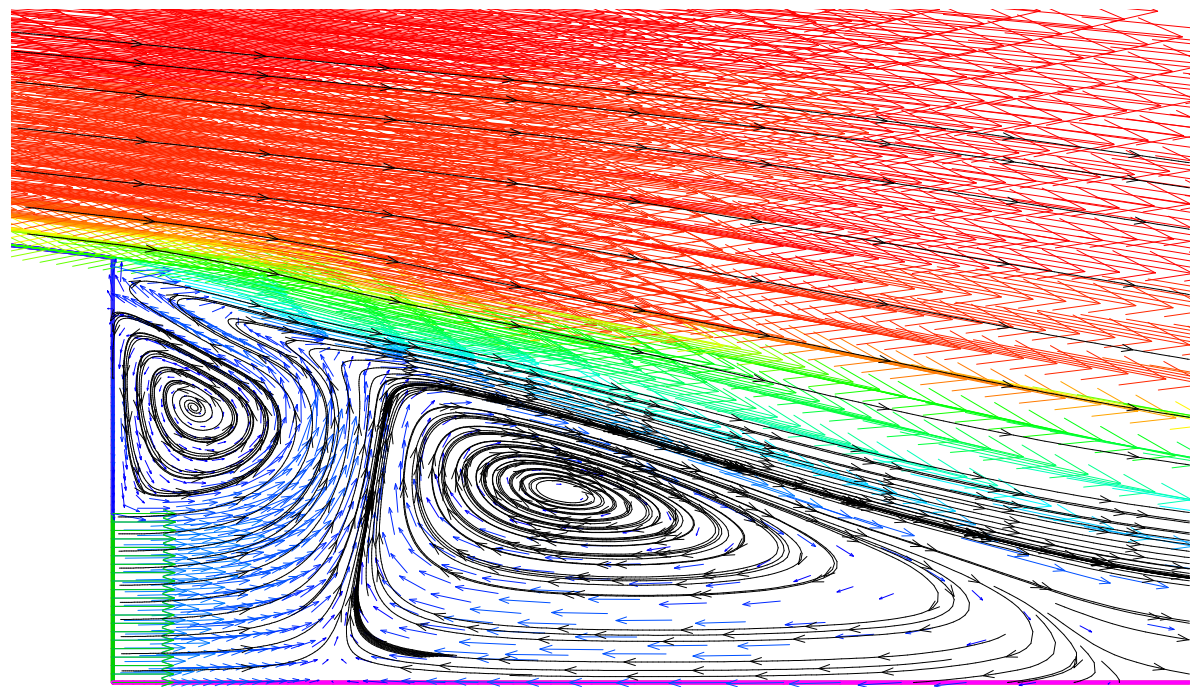

Figure 6: Streamlines in base region at $M=3.0$.

adequate job in predicting viscous layer quantities.

Figure 8 shows the pressure distributions on the wall of two cases with the boattail angle of $\theta=0^{\circ}$ and $\theta=7^{\circ}$. In this figure the present pressure distributions are compared with the wind tunnel test data reported by Schiff [15]. The good agreement of the present pressure distribution with experimental data shows that the drag has been calculated with a high degree of accuracy.

\subsection{Drag Optimization}

Now that reasonable flow solutions have been computed for the case of an axisymmetric body with a boattail afterbody with base bleed, the optimization routine may be implemented in order to determine the boattail angle which minimizes the drag. A consideration when completing this optimization is to reduce the overall computer time, if the optimization routine were allowed to 

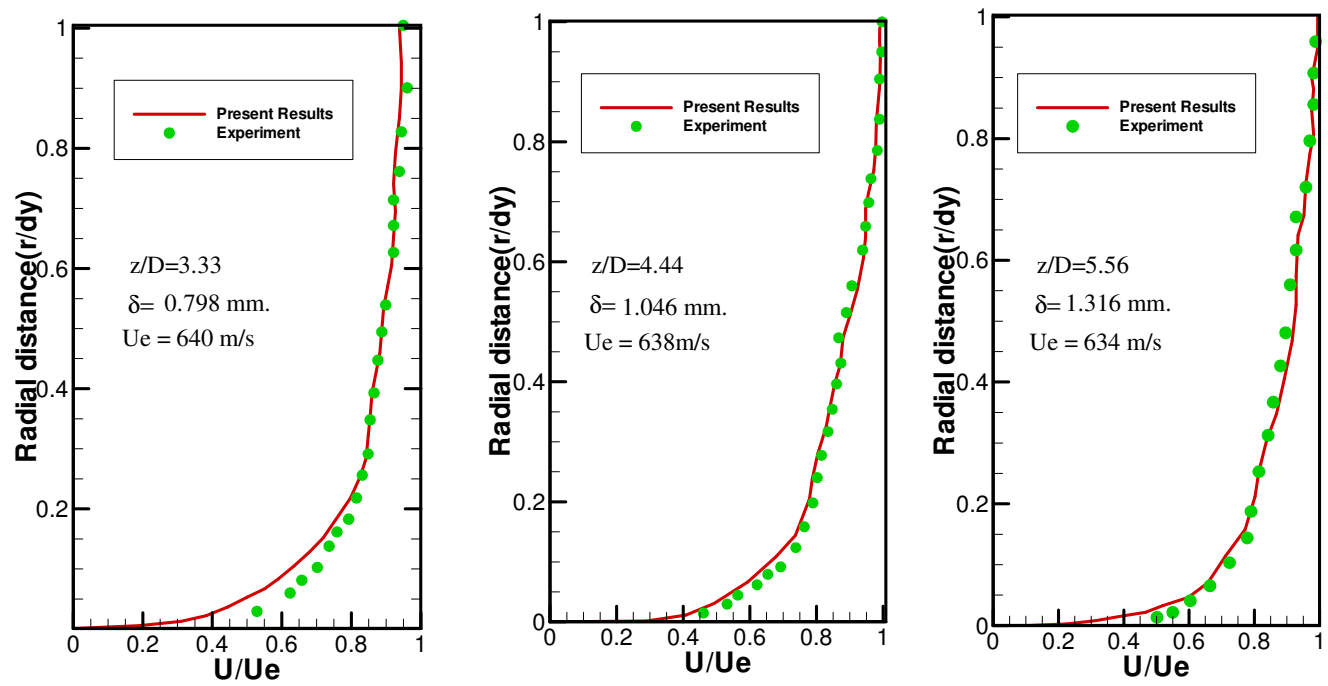

Figure 7: Boundary layer profiles for $M=3.0, \theta=0$.

compute 5 to 10 configurations to full convergence starting from free stream conditions, the computer time would be quite high. To reduce the computational time of the problem, the code was allowed to utilize the previously calculated flow field information as initial conditions for the next case. This resulted in the computational time being reduced to one third for each case. The Golden Section optimization method was allowed to choose the boattail angles for which it required objective function information (drag). The algorithm searched through a pattern of boattail angle as shown in Figure 9. Note the intelligent search path, which utilizes previously obtained information. The algorithm found, in 8 iterations with better than $1 \%$ accuracy, that the minimum value of the drag coefficient was achieved by a boattail angle of $7.9^{\circ}$ for no base bleed and $6.2^{\circ}$ with base bleed. The presence of base bleed decreases the drag coefficient from 0.307 to 0.21 . This is due to the flow pattern and the structure of vortices at the base region. 

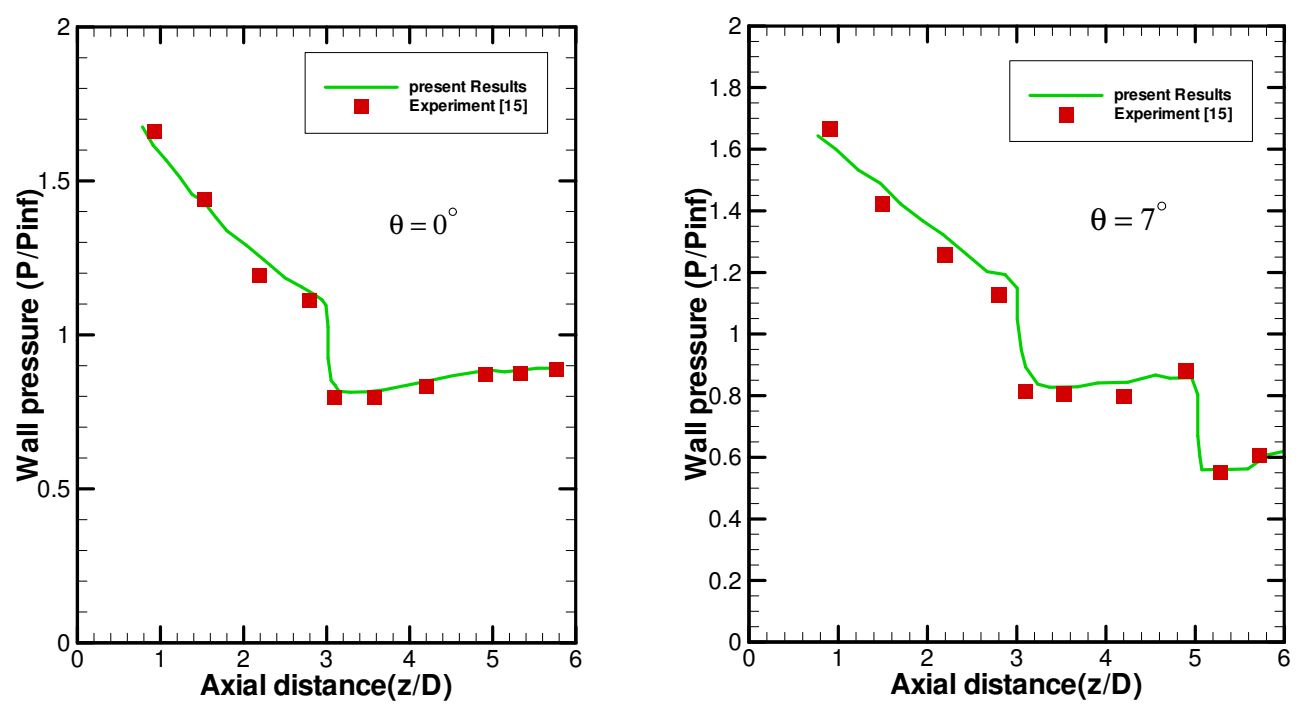

Figure 8: Pressure distribution along the wall for $M=3.0, \operatorname{Re}=6.5 \times 10^{6}$. 

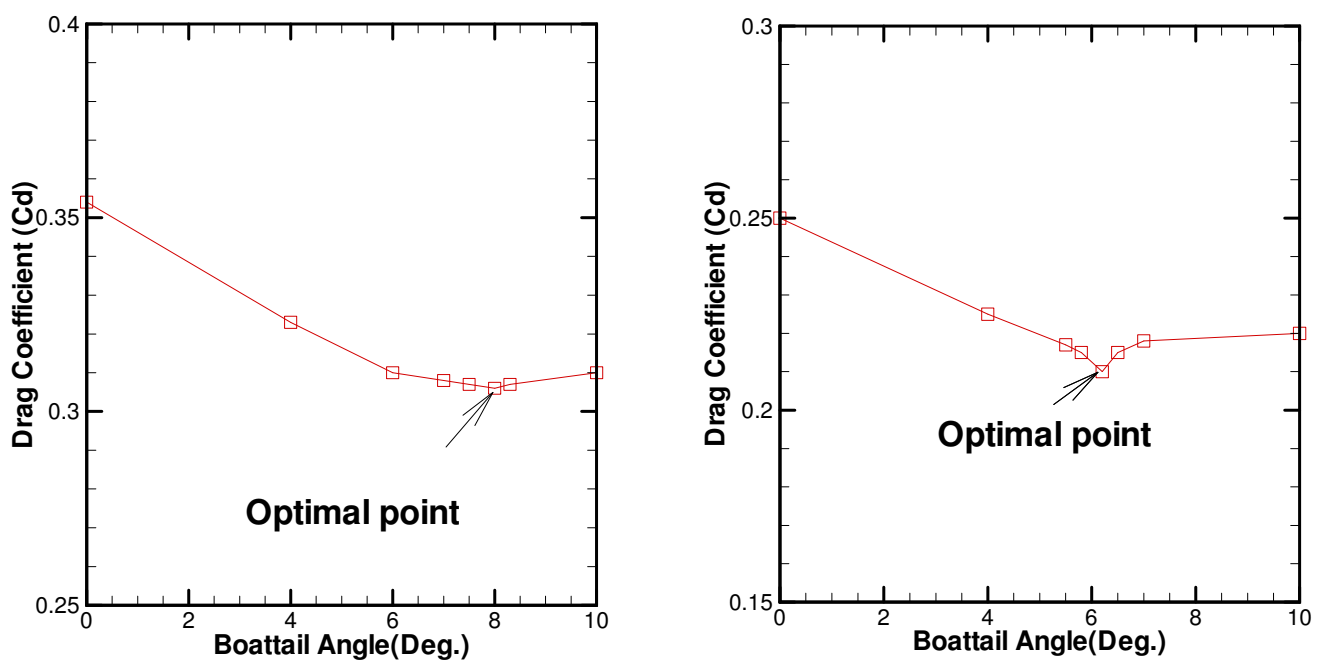

Figure 9: Optimization search path for $M=3.0, \operatorname{Re}=6.5 \times 10^{6}$ : (a) without base bleed; (b) with base bleed. 


\section{Conclusions}

The Kinetic Flux Vector Splitting Method was applied to discretize the Navier-Stokes equations in conjunction with the $k-\epsilon$ turbulence model. The numerical predictions were performed for a complete axisymmetric body with a secant-ogive forebody, cylindrical afterbody, conical boattail, with and without base bleed. The Golden Section search method was used to optimize the boattail angle for minimum drag. The optimum angle was $7.9^{\circ}$ for no basebleed and $6.2^{\circ}$ with base bleed. The prediction of surface pressure compares well with available experimental results. Comparison of viscous layer profiles with available experimental data shows that the $k$ - $\epsilon$ turbulence model adequately describes the viscous features of the flow. Also, the qualitative features of the flow (shock waves, expansion fans, and recirculation region) are well predicted. The lack of experimental data containing flowfield information (such as viscous layer profiles, turbulent quantities, and flow visualization of shocks and expansion fans) for supersonic flow geometries is a great hindrance to validating a numerical code. Nietubicz [13] found that there are virtually no supersonic experiments where flowfield quantities have been measured in the base region. An increased effort needs to be made to return to the wind tunnels and take an in-depth look at the fluid dynamic processes which occur in the flow around bodies at supersonic speeds.

\section{References}

[1] R. M. Cumming, H. Y. Yang, Y. H. Oh, Supersonic, turbulent flow computation and drag optimization for axisymmetric afterbodies, Computers and Fluids, Vol. 24, No. 4, pp. 487-507, 1995. http://www.sciencedirect.com/science/journal/00457930 C1072, C1073, C1075, C1076, C1077 
[2] G. S. Deiwert, Numerical simulation of three dimensional boattail afterboady flow fields, AIAA J., 19, 582-588, 1981. http://pdf .aiaa.org/jaPreview/AIAAJ/1981/PVJAPRE50980.pdf C1071

[3] G. S. Deiwert, A computational investigation of supersonic axisymmetric flow over boattails containing a centered propulsive jet, AIAA J., 22, 1358-1365, 1984.

http://pdf . aiaa.org/jaPreview/AIAAJ/1984/PVJAPRE48576.pdf C1071

[4] G. S. Deiwert, H. Rothmund, Three dimensional flow over a conical afterbody containing a centered propulsive jet: a numerical simulation, AIAA Paper 1983-1709, 1983.

http://pdf.aiaa.org/preview/1983/PV1983_1709.pdf C1071

[5] A. D. Gosman, E. E. Khalil, J. H. Whitelaw, The Calculation of two-dimensional turbulent recirculating flows, Springer Berlin, 1979. C1071

[6] C. Hah, B. Lakshminarayana, The prediction of two and three dimensional asymmetric turbulent wakes - a comparison of the performance of three turbulence models for the effects of streamline curvature and rotation, AIAA Paper 1979-1561, 1979. C1071

[7] T. L. Holst, Numerical solution of axisymmetric boattail fields with plume simulators, AIAA Paper 1977-224, 1977.

http://pdf.aiaa.org/preview/1977/PV1977_224.pdf C1071

[8] A. G. Hutton, R. M. Smith, A study of two-equation turbulence model for axsisymmetric recirculation flows, Proceedings Of the Third International Conference on Numerical methods in laminar and turbulent flows, 233-247, Pineridge press, Swansea, Wales, 1983. http://adsabs.harvard.edu/abs/1983nmlt.proc..233H C1071 
[9] J. C. Mandal, S. M. Deshpande, Kinetic flux vector splitting for Euler equations, Computers and Fluids, Vol. 23, No. 2, 1994. http://dx.doi.org/10.1016/0045-7930(94)90050-7 C1073

[10] N. N. Mansour, J. Kim, P. Moin, Computation of turbulent flows over a back facing step, NASA Technical Memorandum 85851, 1983. C1071

[11] A. G. Mikhail, W. L. Hankey, J. S. Shang, Computation of a supersonic flow past an axisymmetric nozzle boattail with jet exhaust, AIAA J., 18, 869-875, 1980.

http://pdf .aiaa.org/jaPreview/AIAAJ/1980/PVJAPRE50828.pdf C1071

[12] S. N. Murthy, J. R. Osborn, Base flow phenomena with and without injection: experimental results, theories, and bibliography, in Aerodynamics of base combustion, Vol. 40 of Progress in astronautics and aeronautics, 7-210, AIAA, New York, 1976.

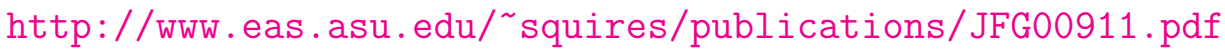
C1071

[13] C. J. Nietubicz, Current CFD efforts in projectile aerodynamics NASA CFD validation workshop, Moffett field, CA, 14-16 July, 1987. C1083

[14] Y. H. Oh, J. E. Harris, Numerical solution of three-dimensional free turbulent shear flows, Proc. of Symposium On Turbulent Shear Flows, 18-20 April 1977, Penn State, University Park, 1977. C1071

[15] L. B. Schiff, W. B. Sturck, Numerical simulation of steady supersonic flow over an ogive cylinder boattail body, U.S. Army ballistic research laboratory Report 2363, September, 1981. http://pdf.aiaa.org/preview/1980/PV1980_66.pdf C1078, C1079 